\title{
Maximum principle and symmetry for minimal hypersurfaces in $\mathbb{H}^{n} \times \mathbb{R}$
}

\author{
BARbara Nelli, RicARdo SA EARP AND ERIC TOUbianA
}

\begin{abstract}
The aim of this work is to study how the asymptotic boundary of a minimal hypersurface in $\mathbb{H}^{n} \times \mathbb{R}$ determines the behavior of the hypersurface at finite points, in several geometric situations.

Mathematics Subject Classification (2010): $53 \mathrm{~A} 10$ (primary); 53C42 (secondary).
\end{abstract}

\section{Introduction}

In this article we discuss how, in several geometric situations, the shape at infinity of a minimal surface in $\mathbb{H}^{2} \times \mathbb{R}$ determines the shape of the surface itself. A beautiful theorem in minimal surfaces theory is the Schoen's characterization of the catenoid [13]. It can be stated as follows. Let $M \subset \mathbb{R}^{3}$ be a complete immersed minimal surface with two annular ends. Assume that each end is a graph, then $M$ is a catenoid. On the other hand, there exists a complete minimal annulus immersed in a slab of $\mathbb{R}^{3}$ [7]. A characterization of the catenoid in hyperbolic space, assuming regularity at infinity, was established by G. Levitt and H. Rosenberg in [6]. In a joint work with L. Hauswirth [4], the authors of the present article proved a Schoen-type theorem in $\mathbb{H}^{2} \times \mathbb{R}$, in the class of finite total curvature surfaces.

In order to state our results we must recall the notion of asymptotic boundary of a surface. We denote the ideal boundary of $\mathbb{H}^{2} \times \mathbb{R}$ by $\partial_{\infty}\left(\mathbb{H}^{2} \times \mathbb{R}\right)$, (see [3] for a definition). As we usually work in the disk model $D_{1}$ for $\mathbb{H}^{2}, \partial_{\infty}\left(\mathbb{H}^{2} \times \mathbb{R}\right)$ is naturally identified with the cylinder $\partial D_{1} \times \mathbb{R}$ joined with the endpoints of all the non horizontal geodesic of $\mathbb{H}^{2} \times \mathbb{R}$. The asymptotic boundary of a surface $M$ in $\mathbb{H}^{2} \times \mathbb{R}$ is the set of the limit points of $M$ in $\partial_{\infty}\left(\mathbb{H}^{2} \times \mathbb{R}\right)$ with respect to the Euclidean topology of $D_{1} \times \mathbb{R}$. The asymptotic boundary of the surface $M$ will be denoted by $\partial_{\infty} M$, while the usual (finite) boundary of $M$ will be denoted by $\partial M$. Analogous notions of boundaries hold in higher dimension. We would like to mention the fact that, in view of our results, we mainly need assumptions about the points of $\partial_{\infty} M$ lying on $\partial_{\infty} \mathbb{H}^{2} \times \mathbb{R}$.

The authors were partially supported by CNPq and FAPERJ of Brasil.

Received November 11, 2012; accepted in revised version March 16, 2013. 
Our first result is a new Schoen-type theorem in $\mathbb{H}^{2} \times \mathbb{R}$. Namely, we replace Schoen's assumption that each end is a graph by the assumption that each end is a vertical graph whose asymptotic boundary is a copy of the asymptotic boundary of $\mathbb{H}^{2}$ (Theorem 2.3).

Our second result is a maximum principle in a vertical (closed) halfspace. Assume that $M$ is a minimal surface, possibly with finite boundary, properly immersed in $\mathbb{H}^{2} \times \mathbb{R}$ and that the boundary of $M$, if any, is contained in the closure of a vertical halfspace $P_{+}$. Assume further that the points at finite height of the asymptotic boundary of $M$ are contained in the asymptotic boundary of the halfspace $P_{+}$. Then $M$ is entirely contained in the halfspace $P_{+}$, unless $M$ is contained in the vertical halfplane $\partial P_{+}$(Theorem 3.2).

Then we generalize our results to higher dimensions. Theorem 2.3 and Theorem 3.2 in higher dimension are analogous to the 2-dimensional case. In order to generalize Theorem 2.3, we first need to give a characterization of the $n$-catenoid analogous to that of the 2-dimensional case (Theorem 4.3, see also [2]). Moreover in the higher dimensional case, it is worthwhile to state some interesting consequences of our results. Let $S_{\infty}$ be a closed set contained in an open slab of $\partial_{\infty} \mathbb{H}^{n} \times \mathbb{R}$ with height equal to $\pi /(n-1)$ such that the projection of $S_{\infty}$ on $\partial_{\infty} \mathbb{H}^{n} \times\{0\}$ omits an open subset. We prove that there is no properly immersed minimal hypersurface $M$ whose asymptotic boundary is $S_{\infty}$ (Theorem 4.6-(2)).

Finally we prove an Asymptotic Theorem (Theorem 4.7), that implies the following non-existence result. There is no horizontal minimal graph over a bounded strictly convex domain, see [10, Equation (3)], given by a positive function $g$ continuous up to the boundary, taking zero boundary value data (Remark 4.9).

ACKNOWLEDGEMENTS. The first and the third authors wish to thank the Departamento de Matemática da PUC-Rio for the kind hospitality. The first and the second authors wish to thank the Laboratoire Géométrie et Dynamique de l'Institut de Mathématiques de Jussieu for the kind hospitality. Finally we would like to thank the referee for carefully reading our manuscript and for giving constructive comments.

\section{A characterization of the catenoid in $\mathbb{H}^{2} \times \mathbb{R}$}

We are going to prove the characterization of the catenoid presented in the Introduction.

For any fixed $t$, the surface $\mathbb{H}^{2} \times\{t\}$ is a complete totally geodesic surface called slice. For any $s \in \mathbb{R}$, we denote by $\Pi_{s}$ the slice $\mathbb{H}^{2} \times\{s\}$ and we set $\Pi_{s}^{+}=\left\{(p, t) \mid p \in \mathbb{H}^{2}, t>s\right\}$ and $\Pi_{s}^{-}=\left\{(p, t) \mid p \in \mathbb{H}^{2}, t<s\right\}$. For simplicity $\Pi$ stands for $\Pi_{0}$.

Lemma 2.1. Let $\Gamma^{+}$and $\Gamma^{-}$be two Jordan curves in $\partial_{\infty} \mathbb{H}^{2} \times \mathbb{R}$ which are vertical graphs over $\partial_{\infty} \mathbb{H}^{2} \times\{0\}$ and such that $\Gamma^{+} \subset \partial_{\infty} \Pi^{+}$and $\Gamma^{-} \subset \partial_{\infty} \Pi^{-}$. Assume 
that $\Gamma^{-}$is the symmetry of $\Gamma^{+}$with respect to $\Pi$. Let $M \subset \mathbb{H}^{2} \times \mathbb{R}$ be an immersed, connected, complete minimal surface with two ends $E^{+}$and $E^{-}$. Assume that each end is a vertical graph and that $\partial_{\infty} M=\Gamma^{+} \cup \Gamma^{-}$, that is $\partial_{\infty} E^{+}=\Gamma^{+}$and $\partial_{\infty} E^{-}=\Gamma^{-}$. Then $M$ is symmetric with respect to $\Pi$. Furthermore, each part $M \cap \Pi^{ \pm}$is a vertical graph and $M$ is embedded.

Proof. For any $t>0$ we set $M_{t}^{+}=M \cap \Pi_{t}^{+}$. We denote by $M_{t}^{+*}$ the symmetry of $M_{t}^{+}$with respect to the slice $\Pi_{t}$. Furthermore, we denote by $t^{+}$the highest $t$ coordinate of $\Gamma^{+}$. Since $\partial_{\infty} M=\Gamma^{+} \cup \Gamma^{-}$, then $M \cap \Pi_{t^{+}}=\emptyset$, by the maximum principle.

We denote by $E^{+}$the end of $M$ whose asymptotic boundary is $\Gamma^{+}$. As $E^{+}$is a vertical graph, there exists $\varepsilon>0$ such that $M_{t^{+}-\varepsilon}^{+}$is a vertical graph, then we can start Alexandrov reflection [1].

We keep doing Alexandrov reflection with $\Pi_{t}$, doing $t \searrow 0$. By applying interior or the boundary maximum principle, we get that, for $t>0$, the surface $M_{t}^{+*}$ stays above $M_{t}^{-}$. Therefore we get that $M_{0}^{+}$is a vertical graph and that $M_{0}^{+*}$ stays above $M_{0}^{-}$.

Doing Alexandrov reflection with slices coming from below, one has that $M_{0}^{-}$ is a vertical graph and that $M_{0}^{-*}$ stays below $M_{0}^{+}$, henceforth we get $M_{0}^{+*}=M_{0}^{-}$. Thus $M$ is symmetric with respect to $\Pi$ and each component of $M \backslash \Pi$ is a graph. Therefore we can show, as in the proof of [13, Theorem 2], that the whole surface $M$ is embedded. This completes the proof.

Definition 2.2. A vertical plane is a complete totally geodesic surface $\gamma \times \mathbb{R}$ where $\gamma$ is any complete geodesic of $\mathbb{H}^{2}$.

Theorem 2.3. Let $M \subset \mathbb{H}^{2} \times \mathbb{R}$ be an immersed, connected, complete minimal surface with two ends. Assume that each end is a vertical graph whose asymptotic boundary is a copy of $\partial_{\infty} \mathbb{H}^{2}$. Then $M$ is rotational, hence $M$ is a catenoid.

Proof. Up to a vertical translation, we can assume that the asymptotic boundary is symmetric with respect to the slice $\Pi$. We use the same notations as in the proof of Lemma 2.1. We know from Lemma 2.1 that $M$ is symmetric with respect to $\Pi$ and that $M_{0}^{+}$and $M_{0}^{-}$are vertical graphs. Therefore, at any point of $M \cap \Pi$ the tangent plane of $M$ is orthogonal to $\Pi$.

We have $\partial_{\infty} M=\partial_{\infty} \mathbb{H}^{2} \times\left\{t_{0},-t_{0}\right\}$ for some $t_{0}>0$. Since $M$ is embedded, $M$ separates $\mathbb{H}^{2} \times\left[-t_{0}, t_{0}\right]$ into two connected components. We denote by $U_{1}$ the component whose asymptotic boundary is $\partial_{\infty} \mathbb{H}^{2} \times\left[-t_{0}, t_{0}\right]$ and by $U_{2}$ the component such that $\partial_{\infty} U_{2}=\partial_{\infty} \mathbb{H}^{2} \times\left\{t_{0},-t_{0}\right\}$. Let $q_{\infty} \in \partial_{\infty} \mathbb{H}^{2}$ and let $\gamma \subset \mathbb{H}^{2}$ be an oriented geodesic issuing from $q_{\infty}$, that is $q_{\infty} \in \partial_{\infty} \gamma$. Let $q_{0} \in \gamma$ be any fixed point. For any $s \in \mathbb{R}$, we denote by $P_{s}$ the vertical plane orthogonal to $\gamma$ passing through the point of $\gamma$ whose oriented distance from $q_{0}$ is $s$. We suppose that $s<0$ for any point in the half-geodesic $\left(q_{0}, q_{\infty}\right)$. For any $s \in \mathbb{R}$, we call $M_{s}(l)$ the part of $M \backslash P_{s}$ such that $\left(q_{\infty}, t_{0}\right),\left(q_{\infty},-t_{0}\right) \in \partial_{\infty} M_{s}(l)$ and let $M_{s}^{*}(l)$ be the reflection of $M_{s}(l)$ about $P_{s}$. We denote by $M_{s}(r)$ the other part of $M \backslash P_{s}$ and by $M_{s}^{*}(r)$ its reflection about $P_{s}$. 
It will be clear from the following two Claims, why we can start Alexandrov reflection with respect to the vertical planes $P_{s}$ and obtain the result. By assumption, there exists $s_{1}<0$ such that, for any $s<s_{1}$, the part $M_{s}(l)$ has two connected components and both of them are vertical graphs. We deduce that $\partial M_{s}(l)$ has two (symmetric) connected components, each one being a vertical graph. We recall that $\Pi^{+}:=\{t>0\}$ and $\Pi^{-}:=\{t<0\}$.

Claim 1. For any $s<s_{1}$, we have that $M_{s}^{*}(l) \cap \Pi^{+}$stays above $M_{s}(r)$ and $M_{s}^{*}(l) \cap$ $\Pi^{-}$stays below $M_{s}(r)$. Consequently $M_{s}^{*}(l) \subset U_{2}$ for any $s<s_{1}$.

Observe that $M_{s}^{*}(l) \cap \Pi^{+}$and $M_{s}(r) \cap \Pi^{+}$have the same asymptotic boundary and that $\partial\left(M_{s}^{*}(l) \cap \Pi^{+}\right)=\partial M_{s}(r) \cap \Pi^{+}$. Therefore the asymptotic and finite boundaries of $M_{s}^{*}(l)+(0,0, t), t>0$, are above the asymptotic and finite boundaries of $M_{s}(r)$. Hence $M_{s}^{*}(l)+(0,0, t), t>0$, is above $M_{s}(r)$ by the maximum principle, which ensures that the whole $M_{s}^{*}(l) \cap \Pi^{+}$stays above $M_{s}(r)$ for any $s<s_{1}$, as desired. The proof of the other assertion is analogous. Then, Claim 1 is proved.

We now set

$$
\sigma=\sup \left\{s \in \mathbb{R} \mid M_{t}^{*}(l) \cap \Pi^{+} \text {stays above } M_{t}(r) \cap \Pi^{+} \text {for any } t \in(-\infty, s)\right\} .
$$

Claim 2. We have $M_{\sigma}^{*}(l)=M_{\sigma}(r)$. Thus, given a geodesic $\gamma \subset \mathbb{H}^{2}$, there exists a vertical plane $P_{\sigma}$ orthogonal to $\gamma$ such that $M$ is symmetric with respect to $P_{\sigma}$.

Note that we also have

$$
\sigma=\sup \left\{s \in \mathbb{R} \mid M_{t}^{*}(l) \subset U_{2} \text { for any } t \in(-\infty, s)\right\} .
$$

In order to prove Claim 2, we first establish the following fact.

Assertion. For any such that $M_{s}^{*}(l) \cap \Pi \subset U_{2}$, then $M_{s}^{*}(l) \subset U_{2}$.

As $M$ is symmetric with respect to $\Pi$ the intersection $M \cap \Pi$ is constituted of a finite number of pairwise disjoint Jordan curves $C_{1}, \ldots, C_{k}$. Since $M \cap \Pi^{+}$is a vertical graph we deduce

$$
\left(C_{j} \times \mathbb{R}\right) \cap M=C_{j} \quad \text { for any } j=1, \ldots, k .
$$

Moreover, since $M$ is connected and symmetric about $\Pi$, we get that $M \cap \Pi^{+}$is connected. Let $D_{j} \subset \Pi$ be the Jordan domain bounded by $C_{j}, j=1, \ldots, k$. Noticing that:

- $\left(M \cap \Pi^{+}\right) \backslash\left(\bar{D}_{j} \times \mathbb{R}\right) \neq \emptyset$;

- $M \cap \Pi^{+}$is connected;

- $M \cap\left(C_{j} \times \mathbb{R}\right)=C_{j}$;

- $\partial_{\infty} M \cap \Pi^{+}=\partial_{\infty} \mathbb{H}^{2} \times\left\{t_{0}\right\}$; 
we get that $\left(M \cap \Pi^{+}\right) \cap\left(D_{j} \times \mathbb{R}\right)=\emptyset, j=1, \ldots, k$. Hence, $D_{i} \cap D_{j}=\emptyset$ for any $i \neq j$. Therefore, $M \cap \Pi^{+}$is a vertical graph over $\Pi \backslash \cup D_{i}$. By the previous facts, we deduce that $M_{s}^{*}(l) \cap \Pi \subset \cup \bar{D}_{i}$. This implies that $\partial\left(M_{s}^{*}(l) \cap \Pi^{+}\right) \cap \Pi \subset \cup \bar{D}_{i}$. Consequently we get that $\partial\left(M_{s}^{*}(l) \cap \Pi^{+}\right)+(0,0, \varepsilon)$ stays above $M$ for any $\varepsilon>$ 0 . Observe that the asymptotic boundary of $\partial\left(M_{s}^{*}(l) \cap \Pi^{+}\right)+(0,0, \varepsilon)$ also stays above $\partial_{\infty} M$. We conclude by the maximum principle that the vertical translation $\left(M_{s}^{*}(l) \cap \Pi^{+}\right)+(0,0, \varepsilon)$ stays above $M$ for any $\varepsilon>0$. This proves the Assertion.

Let us continue the proof of Claim 2. The definition of $\sigma$ implies that $M_{\sigma+\varepsilon}^{*}(l) \cap$ $U_{1} \neq \emptyset$, for $\varepsilon$ small enough. We deduce from the Assertion that $M_{\sigma+\varepsilon}^{*}(l) \cap \Pi$ is not contained in $U_{2}$ for any small enough $\varepsilon>0$. Hence we infer that $M_{\sigma}^{*}(l) \cap \Pi$ and $M_{\sigma}(r) \cap \Pi$ are tangent at an interior or boundary point lying in some Jordan curve $C_{j}$ contained in $M \cap \Pi$. Since $M_{\sigma}^{*}(l) \subset \bar{U}_{2}, M_{\sigma}(r) \subset \partial U_{2}$ and the tangent plane of $M$ is vertical along $M \cap \Pi$, we are able to apply the maximum principle (possibly with boundary) to conclude that $M_{\sigma}^{*}(l)=M_{\sigma}(r)$, that is $P_{\sigma}$ is a plane of symmetry of $M$. This proves Claim 2 .

For any $\alpha \in(0, \pi / 2]$ consider a continuous family of vertical planes making an angle $\alpha$ with $P_{\sigma}$, generated by hyperbolic translations along the horizontal geodesic $P_{\sigma} \cap \Pi$. Observe that the vertical planes of this family are not anymore orthogonal to a fixed horizontal geodesic. Nevertheless, the reflections with respect to any to those vertical planes keep globally unchanged the asymptotic boundary of $M$. Therefore we can perform Alexandrov reflection with this family of planes and, as before, we find a vertical plane of symmetry of $M$, say $P^{\alpha}$. Hence $M$ is invariant by the rotation of angle $2 \alpha$ around the vertical geodesic $P^{\alpha} \cap P_{\sigma}$. Choosing an angle $\alpha$ such that $\pi / \alpha$ is not rational, we find that $M$ is invariant by rotation around the axis $P^{\alpha} \cap P_{\sigma}$. This concludes the proof of Theorem 2.3.

Remark 2.4. For any integer $n$, there exists a minimal surface in $\mathbb{H}^{2} \times \mathbb{R}$ which is a vertical graph, whose asymptotic boundary is a copy of $\partial_{\infty} \mathbb{H}^{2}$ and whose finite boundary is constituted of $n$ smooth Jordan curves in the slice $\Pi$, see [11, Theorem 5.1]. In the same article the second and the third author asked about the existence of such graphs with two boundary curves in $\Pi$ cutting orthogonally the slice $\Pi$. Theorem 2.3 implies that the answer to this question is negative.

\section{Maximum principle in a vertical halfspace of $\mathbb{H}^{2} \times \mathbb{R}$}

In this section we prove a maximum principle in a vertical halfspace. More precisely, we prove that, under some geometric assumptions, the behavior of the asymptotic boundary of $M$ at finite height, determines the behaviour of $M$.

Definition 3.1. We call a vertical halfspace any of the two components of $\left(\mathbb{H}^{2} \times\right.$ $\mathbb{R}) \backslash P$, where $P$ is a vertical plane.

Theorem 3.2. Let $M$ be a minimal surface, possibly with finite boundary, properly immersed in $\mathbb{H}^{2} \times \mathbb{R}$. Let $P$ be a vertical plane and let $P_{+}$be one of the two halfspaces determined by $P$. If $\partial M \subset \overline{P_{+}}$and $\partial_{\infty} M \cap\left(\partial_{\infty} \mathbb{H}^{2} \times \mathbb{R}\right) \subset \partial_{\infty} P_{+}$, then $M \backslash \partial M \subset P_{+}$, unless $M \subset P$. 
For the proof of Theorem 3.2 we need to consider the one-parameter family of surfaces $M_{d}, d>0$, that have origin in [8, Section 4] and whose geometry is described in [11, Proposition 2.1]. This family of surfaces was already used, for example, in [9, Example 2.1]. We first describe the asymptotic boundary of $M_{d}$, for $d>1$. Consider a horizontal geodesic $\gamma$ in $\mathbb{H}^{2}$, with asymptotic boundary $\{p, q\}$ and let $\alpha$ be the closure of a connected component of $\left(\partial_{\infty} \mathbb{H}^{2} \times\{0\}\right) \backslash(\{p, q\} \times\{0\})$. Let

$$
H(d)=\int_{\cosh ^{-1}(d)}^{+\infty} \frac{d}{\sqrt{\cosh ^{2} u-d^{2}}} d u, d>1
$$

be the positive number defined in [11, (1)]. Notice that $\lim _{d \rightarrow 1} H(d)=+\infty$ and $\lim _{d \rightarrow+\infty} H(d)=\pi / 2$.

Let $\alpha_{d}$ in $\partial_{\infty} \mathbb{H}^{2} \times\{H(d)\}$ and $\alpha_{-d}$ in $\partial_{\infty} \mathbb{H}^{2} \times\{-H(d)\}$ be the two curves that project vertically onto $\alpha$. Let $L_{d}, R_{d}$ be two vertical segments in $\partial_{\infty} \mathbb{H}^{2} \times \mathbb{R}$ of height $2 H(d)$ such that the curve $L_{d} \cup \alpha_{d} \cup R_{d} \cup \alpha_{-d}$ is a closed simple curve. Then $\partial_{\infty} M_{d}=L_{d} \cup \alpha_{d} \cup R_{d} \cup \alpha_{-d}$. Now we describe the position of $M_{d}$ in the ambient space, for $d>1$. First notice that $M_{d}$ is symmetric about $\mathbb{H}^{2} \times\{0\}$ and it is invariant by any isometry of $\mathbb{H}^{2} \times \mathbb{R}$ that induces a hyperbolic translation along $\gamma$.

Denote by $Q_{\gamma}$ the halfspace determined by $\gamma \times \mathbb{R}$, whose asymptotic boundary contains the curve $\alpha$. Let $\gamma_{d}$ be the curve in $Q_{\gamma} \cap\left(\mathbb{H}^{2} \times\{0\}\right)$ at constant distance $\cosh ^{-1}(d)$ from $\gamma . M_{d}$ contains the curve $\gamma_{d}$. Denote by $Z_{d}$ the closure of the non mean convex side of the cylinder over the curve $\gamma_{d}$. Then, $M_{d}$ is contained in $Z_{d}$ which is contained in $Q_{\gamma}$. Notice that any vertical translation of the surface $M_{d}$ is contained in $Z_{d}$. Moreover, any vertical translation of $M_{d}$ is arbitrarily close to $Q_{\gamma}$ if $d$ is sufficiently close to 1 .

We observe that in the description above, $\gamma$ can be any geodesic of $\mathbb{H}^{2}$.

Proof of Theorem 3.2. The proof is an application of the maximum principle between the surface $M$ and the one-parameter family of surfaces $M_{d}, d>1$. We choose the geodesic $\gamma$, in order to construct the $M_{d}$ 's, as follows. Let $\gamma \subset \mathbb{H}^{2}$ be any geodesic such that

- P1: The halfspace $Q_{\gamma}$ is strictly contained in $\left(\mathbb{H}^{2} \times \mathbb{R}\right) \backslash P_{+}$;

- P2: $\partial_{\infty} \gamma \cap \partial_{\infty} P=\emptyset$.

Now, notice that:

(1) The intersection of $\partial_{\infty} M$ with $\partial_{\infty}\left(\mathbb{H}^{2} \times \mathbb{R}\right) \backslash \partial_{\infty} P_{+}$contains no points at finite height;

(2) The asymptotic boundary of any vertical translation of $M_{d}$ is contained in the asymptotic boundary of $Q_{\gamma} \subset \mathbb{H}^{2} \times \mathbb{R} \backslash P_{+}$.

We claim that $M_{d}$ and $M$ are disjoint for any $d>1$. Indeed, letting $p \longrightarrow q$ (with respect to the Euclidean topology of the arc of circle in $\partial_{\infty} \mathbb{H}^{2}$ between $p$ and $q$ in $\partial_{\infty}\left(\mathbb{H}^{2} \times \mathbb{R} \backslash P_{+}\right)$- recall that $p, q$ are the endpoints of the geodesic $\gamma)$, one has that $M_{d}$ collapses to a vertical segment in $\partial_{\infty} \mathbb{H}^{2} \times \mathbb{R}$. Suppose that, 
when $p \longrightarrow q$, the surfaces $M_{d}$ always have a nonempty intersection with $M$. Then, there would exists a point of the asymptotic boundary of $M$ at finite height in $\partial_{\infty}\left(\mathbb{H}^{2} \times \mathbb{R}\right) \backslash \partial_{\infty} P_{+}$, giving a contradiction with (1). Then, if $M \cap M_{d} \neq$ $\emptyset$, we would obtain a last intersection point between $M$ and some modified $M_{d}$ letting $p \longrightarrow q$, contradicting the maximum principle. Therefore, by the maximum principle, any vertical translation of $M_{d}$ and $M$ are disjoint. Let $d \longrightarrow 1$. By the maximum principle, there is no first point of contact between $M_{d}$ and $M$. As we can apply the maximum principle between any vertical translation of $M_{d}$ and $M$, one has that $M$ is contained in the closed halfspace $\mathbb{H}^{2} \times \mathbb{R} \backslash Q_{\gamma}$ for any geodesic $\gamma$ satisfying the properties $\mathrm{P} 1$ and $\mathrm{P} 2$. Therefore, $M$ is included in the closure of $P_{+}$. Now we have one of the following possibilities:

- Some points of the interior of $M$ touch $\partial P_{+}=P$, then, by the maximum principle, $M \subset P$;

- $M \backslash \partial M$ is contained in the halfspace $P_{+}$.

The result is thus proved.

Let us give a definition, before stating some consequences of Theorem 3.2.

Definition 3.3. We say that $L \subset \partial_{\infty}\left(\mathbb{H}^{2} \times \mathbb{R}\right)$ is a vertical line if $L=\{p\} \times \mathbb{R}$ for some $p \in \partial_{\infty} \mathbb{H}^{2}$. Given vertical consecutive lines $L_{1}, \ldots, L_{k}$ in $\partial_{\infty} \mathbb{H}^{2} \times \mathbb{R}$, we define the set $P\left(L_{1}, \ldots, L_{k}\right)$ as follows. Let $P_{i}$ be the vertical plane such that $\partial_{\infty} P_{i} \cap\left(\partial_{\infty} \mathbb{H}^{2} \times \mathbb{R}\right)=L_{i} \cup L_{i+1}$ (with the convention that $\left.L_{k+1}=L_{1}\right)$. Denote by $\tilde{P}_{i}$ the halfspace determined by the vertical plane $P_{i}$ such that $\bigcup_{j} L_{j} \subset \partial_{\infty} \tilde{P}_{i}$. Then, we set $P\left(L_{1}, \ldots, L_{k}\right):=\cap_{i} \tilde{P}_{i}$.

Corollary 3.4. Let $M$ be a minimal surface, possibly with finite boundary, properly immersed in $\mathbb{H}^{2} \times \mathbb{R}$ and let $\Gamma=\partial_{\infty} M \cap\left(\partial_{\infty} \mathbb{H}^{2} \times \mathbb{R}\right)$. Let $L_{1}, \ldots, L_{k}$ be vertical lines in $\partial_{\infty} \mathbb{H}^{2} \times \mathbb{R}$. If $\Gamma \subset L_{1} \cup \ldots \cup L_{k}$ and $\partial M \subset \overline{P\left(L_{1}, \ldots, L_{k}\right)}$, then $M \backslash \partial M$ is contained in $P\left(L_{1}, \ldots, L_{k}\right)$, unless $M$ is contained in one of the $P_{i}$.

Proof. By Theorem 3.2, $M$ is contained in every halfspace $\tilde{P}_{i}$ determined by the vertical plane $P_{i}$ such that $\bigcup_{j} L_{j} \subset \partial_{\infty} \tilde{P}_{i}$, unless it is contained in one of the $P_{i}$. Hence it is contained in $P\left(L_{1}, \ldots, L_{k}\right)$, by definition, unless it is contained in one of the $P_{i}$.

Corollary 3.5. Let $M$ be a complete minimal surface properly immersed in $\mathbb{H}^{2} \times \mathbb{R}$. Let $P$ be a vertical plane. If $\partial_{\infty} M \cap\left(\partial_{\infty} \mathbb{H}^{2} \times \mathbb{R}\right) \subset \partial_{\infty} P$, then $M=P$.

Proof. By Theorem 3.2, $M$ is contained in the closure of both halfspaces determined by $P$, hence it is contained in $P$. Then $M=P$ because it is complete.

Corollary 3.6. Let $M$ be a complete minimal surface properly immersed in $\mathbb{H}^{2} \times \mathbb{R}$. Suppose that the asymptotic boundary of $M$ is contained in the asymptotic boundary of a totally geodesic plane $S$ of $\mathbb{H}^{2} \times \mathbb{R}$. Then $M=S$. 
Proof. The proof is a simple consequence of the maximum principle and of the previous results. We do it for completeness. First assume that the asymptotic boundary of $M$ is contained in the asymptotic boundary of a slice, say $\{t=0\}$. Then, for $n$ sufficiently large, the slice $\{t=n\}$ is disjoint from $M$. Now, we translate the slice $\{t=n\}$ down. The first contact point, cannot be interior because of the maximum principle, hence $M$ must stay below the slice $\{t=0\}$. One can do the same reasoning with slices coming from the bottom, and $M$ must stay above the slice $\{t=0\}$. Hence $M$ coincides with the slice $\{t=0\}$.

If the asymptotic boundary of $M$ is contained is the asymptotic boundary of a vertical plane, the result follows from Corollary 3.5.

Corollary 3.7. Let $M$ be a minimal surface properly immersed in $\mathbb{H}^{2} \times \mathbb{R}$. Assume that the projection of the asymptotic boundary of $M$ into $\partial_{\infty} \mathbb{H}^{2} \times\{0\}$ omits a closed interval $\alpha$ joining two points $p$ and $q$. Let $\gamma$ be the horizontal geodesic in $\mathbb{H}^{2} \times\{0\}$ whose the asymptotic boundary is $\{p, q\}$ and let $Q_{\gamma}$ be the halfspace determined by $\gamma \times \mathbb{R}$ whose asymptotic boundary contains $\alpha$. Then $M$ is contained in $\mathbb{H}^{2} \times \mathbb{R} \backslash \bar{Q}_{\gamma}$.

Proof. By hypothesis $\partial_{\infty} M \cap\left(\partial_{\infty} \mathbb{H}^{2} \times \mathbb{R}\right)$ is contained in the asymptotic boundary of $\left(\mathbb{H}^{2} \times \mathbb{R}\right) \backslash Q_{\gamma}$. The result follows by Theorem 3.2 with $P_{+}=\left(\mathbb{H}^{2} \times \mathbb{R}\right) \backslash \bar{Q}_{\gamma}$.

Remark 3.8. There exist examples of minimal surfaces with asymptotic boundary equal to two vertical halflines, lines and a curve at finite height, see [8, Equation (32)] and [11, Proposition 2.1 (2)].

\section{Some generalizations to $\mathbb{H}^{n} \times \mathbb{R}$}

Let us recall the construction and the properties of the $n$-catenoids in $\mathbb{H}^{n} \times \mathbb{R}, n \geqslant 3$, established, by P. Bérard and the second author in [2, Proposition 3.2]. Given any $a>0$ we denote by $\left(I_{a}, f(a, \cdot)\right)$, where $I_{a} \subset \mathbb{R}$ is an interval, the maximal solution of the following Cauchy problem:

$$
\left\{\begin{array}{l}
f_{t t}=(n-1)\left(1+f_{t}^{2}\right) \operatorname{coth}(f), \\
f(0)=a>0 \\
f_{t}(0)=0
\end{array}\right.
$$

Theorem 4.1 ([2]). For $a>0$, the maximal solution $\left(I_{a}, f(a, \cdot)\right)$ gives rise to the generating curve $C_{a}$, parametrized by $t \mapsto(\tanh (f(a, t)), t)$, of a complete minimal rotational hypersurface $\mathcal{C}_{a}$ (n-catenoid) in $\mathbb{H}^{n} \times \mathbb{R}$, with the following properties:

(1) The interval $I_{a}$ is of the form $\left.I_{a}=\right]-T(a), T(a)[$, where

$$
T(a)=\sinh ^{n-1}(a) \int_{a}^{\infty}\left(\sinh ^{2 n-2}(u)-\sinh ^{2 n-2}(a)\right)^{-1 / 2} d u ;
$$

(2) $f(a, \cdot)$ is an even function of the second variable;

(3) For all $t \in I_{a}, f(a, t) \geq a$; 
(4) The derivative $f_{t}(a, \cdot)$ is positive on $] 0, T(a)[$, negative on $]-T(a), 0[$;

(5) The function $f(a, \cdot)$ is a bijection from $[0, T(a)[$ onto $[a, \infty[$, with inverse function $\lambda(a, \cdot)$ given by

$$
\lambda(a, \rho)=\sinh ^{n-1}(a) \int_{a}^{\rho}\left(\sinh ^{2 n-2}(u)-\sinh ^{2 n-2}(a)\right)^{-1 / 2} d u ;
$$

(6) The catenoid $\mathcal{C}_{a}$ has finite vertical height $h_{R}(a):=2 T(a)$;

(7) The function $a \mapsto h_{R}(a)$ increases from 0 to $\frac{\pi}{(n-1)}$ when a increases from 0 to infinity. Furthermore, given $a \neq b$, the generating catenaries $C_{a}$ and $C_{b}$ intersect at exactly two symmetric points.

We observe that the $n$-catenoids are properly embedded hypersurfaces. For later use, we need the following result. Although we believe that the result is classical, we give a proof for the sake of completeness. The reader is referred to [5, Chapter VII] or [14, Chapter 9, addendum 3] for the proof of the analogous statement in Euclidean space.

Proposition 4.2. Let $S \subset \mathbb{H}^{n}$ be a finite union of connected, closed and embedded ( $n-1)$-submanifolds $C_{j}, j=1, \ldots, k$, such that the bounded domains whose boundary are the $C_{j}$ are pairwise disjoint. Assume that for any geodesic $\gamma \subset \mathbb{H}^{n}$, there exists a $(n-1)$-geodesic plane $\pi_{\gamma} \subset \mathbb{H}^{n}$ of symmetry of $S$ which is orthogonal to $\gamma$. Then $S$ is a $(n-1)$-geodesic sphere of $\mathbb{H}^{n}$.

Proof. We will do the proof by induction on $n \geqslant 2$. First assume that $n=2$. We may infer from the hypothesis that there exist two geodesics $c_{1}, c_{2} \subset \mathbb{H}^{2}$ of symmetry of the closed curve $S$ intersecting at some point $p \in \mathbb{H}^{2}$ and making an angle $\alpha \neq 0$ such that $\pi / \alpha$ is not rational. For any $q \in S$, denote by $C_{q}$ the circle centered at $p$ passing through $q$. The orbit of $q$ under the rotation centered at $p$, of angle $2 \alpha$, is contained in $S$. Then, being $\pi / \alpha$ not rational, $C_{q}$ is contained in $S$. Let $\widetilde{q} \neq q$ be points of $S$ and let $C_{\widetilde{q}}$ defined as above. If $C_{q} \neq C_{\widetilde{q}}$ then the geodesic disks bounded by $C_{q}$ and $C_{\widetilde{q}}$ are not disjoint, since they have the same center, which contradicts the hypothesis. Consequently, we get $C_{q}=C_{\tilde{q}}$ and we conclude that $S$ is a circle.

Let $n \in \mathbb{N}, n \geqslant 3$. Assume that the statement holds for $k=2, \ldots, n-1$. Let $\pi_{0} \subset \mathbb{H}^{n}$ be a $(n-1)$-geodesic plane of symmetry of $S$.

Claim 1. $S \cap \pi_{0}$ is a $(n-2)$-geodesic sphere of $\pi_{0}$.

Indeed, let $\gamma \subset \pi_{0}$ be a geodesic. By hypothesis there exists a $(n-1)$-geodesic plane $\pi_{\gamma} \subset \mathbb{H}^{n}$ orthogonal to $\gamma$ which is a plane of symmetry of $S$. Since $\pi_{\gamma}$ is orthogonal to $\pi_{0}$, then $S \cap \pi_{0}$ is symmetric about $\pi_{\gamma} \cap \pi_{0}$ (which is a $(n-2)$-geodesic plane of $\left.\pi_{0}\right)$, see [12, Lemme 3.3.15]. As $\pi_{0}$ is a $(n-1)$ hyperbolic space, $S \cap \pi_{0}$ satisfies the assumptions of the statement in $\mathbb{H}^{n-1}$. By the induction hypothesis, we deduce that $S \cap \pi_{0}$ is a $(n-2)$-geodesic sphere of $\pi_{0}$. This proves Claim 1 .

Let $p_{0} \in \pi_{0}$ and $\rho_{0}>0$ be respectively the center and the radius of the $(n-2)$ geodesic sphere $S \cap \pi_{0}$.

Claim 2. Let $\pi_{1} \subset \mathbb{H}^{n}$ be a $(n-1)$-geodesic plane of symmetry of $S$ orthogonal to $\pi_{0}$. Then $S \cap \pi_{1}$ is a $(n-2)$-geodesic sphere of $\pi_{1}$ with center $p_{0}$ and radius $\rho_{0}$. 
Claim 1 yields that $S \cap \pi_{1}$ is a $(n-2)$-geodesic sphere of $\pi_{1}$. Since $\pi_{0}$ and $\pi_{1}$ are orthogonal, then the geodesic sphere $S \cap \pi_{0}$ is symmetric about $\pi_{1}$. Therefore $p_{0} \in \pi_{1}$.

If $n>3$, then $\left(S \cap \pi_{0}\right) \cap \pi_{1}$ is the $(n-3)$-geodesic sphere with center $p_{0}$ and radius $\rho_{0}$ of $\pi_{0} \cap \pi_{1}$ (which is a $(n-2)$ hyperbolic space). If $n=3$, then $\left(S \cap \pi_{0}\right) \cap \pi_{1}$ is constituted of two points whose the distance is $2 \rho_{0}$. In both cases we infer that $\operatorname{diam}_{\mathbb{H}^{n}}\left(S \cap \pi_{1}\right) \geqslant 2 \rho_{0}$ and then the radius of the geodesic sphere $S \cap \pi_{1}$ is $\rho_{1} \geqslant \rho_{0}$. Analogously we can show that $\rho_{0} \geqslant \rho_{1}$. We deduce that $\rho_{1}=\rho_{0}$, that is $S \cap \pi_{0}$ and $S \cap \pi_{1}$ have both center at $p_{0}$ and radius $\rho_{0}$. This proves Claim 2 .

Claim 3. Let $\pi_{2} \subset \mathbb{H}^{n}$ be any $(n-1)$-geodesic plane of symmetry of $S$. Then $S \cap \pi_{2}$ is a $(n-2)$-geodesic sphere of $\pi_{2}$ with center $p_{0}$ and radius $\rho_{0}$.

Since $S$ is symmetric with respect to $\pi_{0}$ and $\pi_{2}, \pi_{0}$ and $\pi_{2}$ are distinct and $S$ is compact, then the $(n-1)$-geodesic planes $\pi_{0}$ and $\pi_{2}$ cannot be disjoint.

Then, we find a third $(n-1)$-geodesic plane $\pi_{3}$ of symmetry of $S$, orthogonal to both $\pi_{0}$ and $\pi_{2}$. Claim 2 implies that $S \cap \pi_{2}$ is a $(n-2)$-geodesic sphere of $\pi_{2}$ with center $p_{0}$ and radius $\rho_{0}$. This proves Claim 3 .

Now we finish the proof of the proposition as follows. Let $p \in S$ and let $\pi \subset \mathbb{H}^{n}$ be any $(n-1)$-geodesic plane passing through $p$ and $p_{0}$. Let $\gamma \subset \mathbb{H}^{n}$ be the geodesic through $p_{0}$ orthogonal to $\pi$. By Claim 2 , there exists a $(n-1)$ geodesic plane $\pi_{\gamma}$ of symmetry of $S$ and orthogonal to $\gamma$. Claim 3 ensures that $p_{0} \in \pi_{\gamma}$, then $\pi_{\gamma}=\pi$. Claim 3 yields also that $S \cap \pi$ is the $(n-2)$-geodesic sphere of $\pi$ with center $p_{0}$ and radius $\rho_{0}$, thus $d_{\mathbb{H}^{n}}\left(p, p_{0}\right)=\rho_{0}$. This shows that $S$ is the $(n-1)$-geodesic sphere of $\mathbb{H}^{n}$ of radius $\rho_{0}$ and center $p_{0}$.

Now we establish a characterization of the $n$-catenoid, that is a generalization to higher dimension of Theorem 2.3.

Theorem 4.3. Let $M \subset \mathbb{H}^{n} \times \mathbb{R}$ be an immersed, connected, complete minimal hypersurface with two ends. Assume that each end is a vertical graph whose asymptotic boundary is a copy of $\partial_{\infty} \mathbb{H}^{n}$. Then $M$ is a $n$-catenoid.

Proof. Up to a vertical translation, we can assume that the asymptotic boundary of $M$ is symmetric with respect to $\Pi:=\mathbb{H}^{n} \times\{0\}$. We set $\Gamma^{+}:=\partial_{\infty} M \cap\{t>0\}$ and recall that $\Gamma^{+}$is a copy of $\partial_{\infty} \mathbb{H}^{n}$. As usual we set $M^{+}:=M \cap\{t>0\}$.

The next claim can be shown in the same fashion as in $\mathbb{H}^{2} \times \mathbb{R}$ (see Lemma 2.1 and the proof of Claim 2 of Theorem 2.3). For this reason we just state it.

Claim. $M$ is symmetric about $\Pi$, and each connected component of $M \backslash \Pi$ is a vertical graph. Moreover, for any geodesic $\gamma \subset \Pi$ there exists a vertical hyperplane $P_{\gamma} \subset \mathbb{H}^{n} \times \mathbb{R}$ orthogonal to $\gamma$ which is a n-plane of symmetry of $M$. Therefore, $\pi_{\gamma}:=P_{\gamma} \cap \Pi$ is $a(n-1)$-plane of symmetry of $\Sigma:=M \cap \Pi$.

Using the result of the Claim we get that $\Sigma$ satisfies the assumptions of Proposition 4.2. Then $\Sigma$ is a $(n-1)$-geodesic sphere of $\Pi$, since $\Pi=\mathbb{H}^{n} \times\{0\}$. Let $\mathcal{C} \subset$ $\mathbb{H} \mathbb{H}^{n} \times \mathbb{R}$ be the catenoid through $\Sigma$ and orthogonal to $\Pi$. We set $\mathcal{C}^{+}:=\mathcal{C} \cap\{t>0\}$. Both $\mathcal{C}^{+}$and $M^{+}$are vertical along their common finite boundary $\Sigma$, hence they 
are tangent along $\Sigma$. Let $t_{\mathcal{C}}$ (respectively $t_{M}$ ) the height of the asymptotic boundary of $\mathcal{C}^{+}$(respectively $M^{+}$). Suppose for example that $t_{\mathcal{C}} \leqslant t_{M}$. Then, lifting upward and downward $M^{+}$, we obtain that $M^{+}$is above $\mathcal{C}^{+}$. Therefore we deduce that $M^{+}=\mathcal{C}^{+}$by applying the boundary maximum principle. The case $t_{M} \leqslant t_{\mathcal{C}}$ is analogous. We conclude that $M=\mathcal{C}$ and the proof is completed.

In order to establish the generalization in higher dimension of Theorem 3.2, we need to state some existence results, established for $n \geqslant 3$, in [2, Theorem 3.8], inspired by [11, Proposition 2.1]. Before stating the theorem, we recall that an equidistant hypersurface is the set of points of $\mathbb{H}^{n} \times\{0\}$ equidistant to a totally geodesic $(n-1)$-hyperbolic submanifold of $\mathbb{H}^{n} \times\{0\}$.

Theorem 4.4 ([2]). There exists a one-parameter family $\left\{\mathcal{M}_{d}, d>1\right\}$ of complete embedded minimal hypersurfaces in $\mathbb{H}^{n} \times \mathbb{R}$ invariant under hyperbolic translations. Moreover $\mathcal{M}_{d}$ consists of the union of two symmetric vertical graphs over the exterior of an equidistant hypersurface in the slice $\mathbb{H}^{n} \times\{0\}$. The asymptotic boundary of $\mathcal{M}_{d}$ is topologically an $(n-1)$-sphere which is homologically trivial in $\partial_{\infty} \mathbb{H}^{n} \times \mathbb{R}$. More precisely, we set:

$S(d)=\cosh (a) \int_{1}^{\infty}\left(t^{2 n-2}-1\right)^{-1 / 2}\left(\cosh ^{2}(a) t^{2}-1\right)^{-1 / 2} d t$, where $d=: \cosh ^{n-1}(a)$.

Then, the asymptotic boundary of $\mathcal{M}_{d}$ consists of the union of two copies of an hemisphere $S_{+}^{n-1} \times\{0\}$ of $\partial_{\infty} \mathbb{H}^{n} \times\{0\}$ in parallel slices $t= \pm S(d)$, glued with the finite cylinder $\partial S_{+}^{n-1} \times[-S(d), S(d)]$. The vertical height of $\mathcal{M}_{d}$ is $2 S(d)$. The height of the family $\mathcal{M}_{d}$ is a decreasing function of $d$ and varies from infinity (when $d \rightarrow 1)$ to $\pi /(n-1)$ (when $d \rightarrow \infty)$.

Actually the family of hypersurfaces $\mathcal{M}_{d}$ is contained in a wider family of hypersurfaces $\left\{\mathcal{M}_{d}, d>0\right\}$ [2]. We observe that all the hypersurfaces $\mathcal{M}_{d}$ are properly embedded. The hypersurfaces $\mathcal{M}_{d}$ are the analogue in higher dimension of the surfaces $M_{d}$ in $\mathbb{H}^{2} \times \mathbb{R}$. Also, as in $\mathbb{H}^{2} \times \mathbb{R}$, by (vertical) hyperplane we mean a complete totally geodesic hypersurface $\Pi \times \mathbb{R}$, where $\Pi$ is any totally geodesic hyperplane of $\mathbb{H}^{n} \times\{0\}$. Moreover, we call a vertical halfspace any component of $\left(\mathbb{H}^{n} \times \mathbb{R}\right) \backslash P$ where $P$ is a vertical hyperplane. Thus, working with the hypersurfaces $\mathcal{M}_{d}$ exactly in the same way as in Theorem 3.2, we obtain the following result:

Theorem 4.5. Let $M$ be a minimal hypersurface properly immersed in $\mathbb{H}^{n} \times \mathbb{R}$, possibly with finite boundary. Let $P$ be a vertical geodesic hyperplane and $P_{+}$one of the two halfspaces determined by $P$. If $\partial M \subset \overline{P_{+}}$and $\partial_{\infty} M \cap\left(\partial_{\infty} \mathbb{H}^{n} \times \mathbb{R}\right) \subset$ $\partial_{\infty} P_{+}$, then $M \backslash \partial M \subset P_{+}$, unless $M \subset P$.

Obviously, the analogues in higher dimension of Corollaries 3.4, 3.5, 3.6 hold as well.

Part (1) of the next theorem is a generalization in higher dimension of Corollary 3.7, while part (2) was proved, for $n=2$, by the second and the third author [11, Corollary 2.2]. 
Theorem 4.6. Let $S_{\infty} \subset \partial_{\infty} \mathbb{H}^{n} \times \mathbb{R}$ be a closed set whose the vertical projection on $\partial_{\infty} \mathbb{H}^{n} \times\{0\}$ omits an open subset $U$.

(1) Let $M$ be a minimal hypersurface properly immersed in $\mathbb{H}^{n} \times \mathbb{R}$ such that $\partial_{\infty} M=S_{\infty}$. Let $Q \subset \mathbb{H}^{n} \times \mathbb{R}$ be a vertical halfspace whose asymptotic boundary is contained in $U \times \mathbb{R}$. Then $M$ is contained in $\mathbb{H}^{n} \times \mathbb{R} \backslash \bar{Q}$;

(2) Assume that $S_{\infty}$ is contained in an open slab whose height is equal to $\frac{\pi}{n-1}$. Then, there is no connected properly immersed minimal hypersurface $M$ in $\mathbb{H}^{n} \times \mathbb{R}$ with asymptotic boundary $S_{\infty}$.

Proof. The first statement is a consequence of Theorem 4.5 and the proof is analogous to that of Corollary 3.7.

Let us prove the second statement. Assume, by contradiction, that there is such a minimal hypersurface $M$ with asymptotic boundary $S_{\infty}$. Then, up to a vertical translation, we can assume that $M$ is contained in the slab $\mathcal{S}:=\left\{\varepsilon<t<\frac{\pi}{n-1}-\varepsilon\right\}$ for some $\varepsilon>0$, and thus $S_{\infty} \subset \partial_{\infty} \mathcal{S}$. Using (1) of the present Theorem and our assumptions, we find an (n-1)-geodesic plane $\pi \subset \mathbb{H}^{n} \times\{0\}$ such that a component $\pi^{+}$of $\mathbb{H}^{n} \times\{0\} \backslash \pi$ satisfies:

(1) $\partial_{\infty} \pi^{+} \subset U$;

(2) $M \cap\left(\pi^{+} \times \mathbb{R}\right)=\emptyset$.

Let $C \subset \mathbb{H}^{n} \times\left(0, \frac{\pi}{n-1}\right)$ be any $n$-catenoid such that a component of its asymptotic boundary stays strictly above $\partial_{\infty} \mathcal{S}$ and the other component stays strictly below $\partial_{\infty} \mathcal{S}$

We take a connected and compact piece $K$ of $C$ such that its boundary lies in the boundary of the slab $\mathcal{S}$. Let $q \in M$ be a point and let $q_{0} \in \mathbb{H}^{n} \times\{0\}$ be the vertical projection of $q$. Let $p_{\infty} \in \partial_{\infty} \pi^{+}$be an asymptotic point. Denote by $\tilde{\gamma} \subset \partial_{\infty} \mathbb{H}^{n} \times\{0\}$ the complete geodesic passing through $q_{0}$ such that $p_{\infty} \in \partial_{\infty} \tilde{\gamma}$. We can translate $K$ along $\widetilde{\gamma}$ such that the translated $K$ is contained in the halfspace $\pi^{+} \times \mathbb{R}$. Now we come back translating $K$ towards $M$ along $\tilde{\gamma}$. Observe that the boundary of the translated copies of $K$ does not touch $M$. Therefore, doing the translations of $K$ along $\tilde{\gamma}$ we find a first interior point of contact between $M$ and a translated copy of $K$. Hence, $M=C$ by the maximum principle, which leads to a contradiction. This completes the proof.

Now we state a generalization of the Asymptotic Theorem proved in [11, Theorem 2.1].

Our result establishes some obstruction for the asymptotic boundary of a properly immersed minimal hypersurface in $\mathbb{H}^{n} \times \mathbb{R}$.

Theorem 4.7 (Asymptotic Theorem). Let $\Gamma \subset \partial_{\infty} \mathbb{H}^{n} \times \mathbb{R}$ be a connected $(n-1)$ submanifold with boundary. Let $\operatorname{Pr}: \partial_{\infty} \mathbb{H}^{n} \times \mathbb{R} \rightarrow \partial_{\infty} \mathbb{H}^{n}$ be the projection on the first factor. Assume that:

(1) There is some point $q_{\infty} \in \partial \operatorname{Pr}(\Gamma)$ such that $q_{\infty} \notin \operatorname{Pr}(\partial \Gamma)$;

(2) $\Gamma \subset \partial_{\infty} \mathbb{H}^{n} \times\left(t_{0}, t_{0}+\frac{\pi}{n-1}\right)$ for some real number $t_{0}$. 
Then there is no properly immersed minimal hypersurface (possibly with finite boundary) $M \subset \mathbb{H}^{n} \times \mathbb{R}$ such that $\partial_{\infty} M=\Gamma$.

Proof. Assume, by contradiction, that there is such a minimal hypersurface $M$. Since $q_{\infty} \in \partial \operatorname{Pr}(\Gamma)$ and $q_{\infty} \notin \operatorname{Pr}(\partial \Gamma)$, there exists a $(n-1)$-geodesic plane $\omega \subset$ $\mathbb{H}^{n} \times\{0\}$ such that a component $\omega^{+}$of $\mathbb{H}^{n} \times\{0\} \backslash \omega$ satisfies:

(1) $q_{\infty} \in \partial_{\infty} \omega^{+}, q_{\infty} \notin \partial_{\infty} \omega$ and $\partial_{\infty} \omega^{+} \cap \operatorname{Pr}(\partial \Gamma)=\emptyset$;

(2) If $M_{0}$ denotes a component of $M \cap\left(\omega^{+} \times \mathbb{R}\right)$ containing $q_{\infty}$ in its asymptotic boundary, then

(a) $M_{0} \subset \mathbb{H}^{n} \times\left(t_{0}, t_{0}+\frac{\pi}{n-1}\right)$ for some real number $t_{0}$;

(b) $\partial M_{0} \subset \omega \times\left(t_{0}+2 \varepsilon, t_{0}-2 \varepsilon+\frac{\pi}{n-1}\right)$ for some $\varepsilon>0$.

Again, since $q_{\infty} \in \partial \operatorname{Pr}(\Gamma)$ and $q_{\infty} \notin \operatorname{Pr}(\partial \Gamma)$, there exists a $(n-1)$-geodesic plane $\pi \subset \mathbb{H}^{n} \times\{0\}$ such that a component $\pi^{+}$of $\mathbb{H}^{n} \times\{0\} \backslash \pi$ satisfies:

(1) $\pi^{+} \subset \omega^{+}$;

(2) $\partial_{\infty} \pi^{+} \cap \operatorname{Pr}(\Gamma)=\emptyset$;

(3) $M_{0} \cap\left(\pi^{+} \times \mathbb{R}\right)=\emptyset$.

Therefore we can find a compact part $K$ of a $n$-catenoid satisfying:

(1) $K$ is connected;

(2) $K \subset \pi^{+} \times\left(t_{0}+\varepsilon, t_{0}-\varepsilon+\frac{\pi}{n-1}\right)$;

(3) $\partial K \subset \mathbb{H}^{n} \times\left\{t_{0}+\varepsilon, t_{0}-\varepsilon+\frac{\pi}{n-1}\right\}$.

We deduce consequently that $M_{0} \cap K=\emptyset$. Then, considering the horizontal translated copies of $K$ and arguing as in the proof of Theorem 4.6, we get a contradiction by the maximum principle, which concludes the proof.

The following result is an immediate consequence of Theorem 4.7:

Corollary 4.8. Let $S_{\infty} \subset \partial_{\infty} \mathbb{H}^{n} \times \mathbb{R}$ be an $(n-1)$-closed continuous submanifold Considering the halfspace model for $\mathbb{H}^{n}$, we can assume that $S_{\infty} \subset \mathbb{R}^{n-1} \times \mathbb{R}$. If $S_{\infty}$ is strictly convex in Euclidean sense, then there is no connected properly immersed minimal hypersurface $M$ in $\mathbb{H}^{n} \times \mathbb{R}$, possibly with finite boundary, with asymptotic boundary $S_{\infty}$.

Remark 4.9. It follows from Corollary 4.8 that there is no horizontal minimal graph in $\mathbb{H}^{n} \times \mathbb{R}$, [10, Equation (3)], given by a positive function $g \in C^{2}(\Omega) \cap$ $C^{0}(\bar{\Omega})$, where $\Omega \subset \mathbb{R}^{n-1} \times \mathbb{R} \subset \partial_{\infty} \mathbb{H}^{n} \times \mathbb{R}$ is a bounded strictly convex domain in Euclidean sense, assuming zero value on $\partial \Omega$.

\section{References}

[1] A. D. AleXANDrov, Uniqueness theorems for surfaces in the large. I, Amer. Math. Soc. Transl. (2) 21 (1962), 341-354.

[2] P. BÉRARD and R. SA EARP, Minimal hypersurfaces in $\mathbb{H}^{n} \times \mathbb{R}$, total curvature and index, arXiv: $0808.3838 v 3$. 
[3] P. Eberlein and B. O’Neill, Visibility manifolds, Pacific J. Math. 46 (1973), 45-109.

[4] L. Hauswirth, B. Nelli, R. Sa EARP and E.Toubiana, A Schoen theorem for minimal surfaces in $\mathbb{H}^{2} \times \mathbb{R}$, Adv. Math. 274 (2015), 199-240.

[5] H. Hopf, "Differential Geometry in the Large", Notes taken by Peter Lax and John W. Gray. With a preface by S. S. Chern, Second edition, with a preface by K. Voss, Lecture Notes in Mathematics, 1000. Springer-Verlag, Berlin, 1989.

[6] G. LEVITT and H. ROSENBERG, Symmetry of constant mean curvature hypersurfaces in hyperbolic space, Duke Math. J. 52 (1985), 53-59.

[7] H. Rosenberg and E. TOUBIANA, A cylindrical type complete minimal surface in a slab of $R^{3}$, Bull. Sci. Math. (2) 111 (1987), 241-245.

[8] R. SA EARP, Parabolic and hyperbolic screw motion in $\mathbb{H}^{2} \times \mathbb{R}$, J. Aust. Math. Soc. 85 (2008), 113-143.

[9] R. SA EARP, Uniqueness of minimal surfaces whose boundary is a horizontal graph and some Bernstein problems in $\mathbb{H}^{2} \times \mathbb{R}$, Math. Z. 273 (2013), 211-217.

[10] R. SA EARP, Uniform a priori estimates for a class of horizontal minimal equations, arXiv: 1205.4375.

[11] R. SA EARP and E. TOUBIANA, An asymptotic theorem for minimal surfaces and existence results for minimal graphs in $\mathbb{H}^{2} \times \mathbb{R}$, Math. Ann. 342 (2008), 309-331.

[12] R. Sa EARP and E. Toubiana, "Introduction à la Géométrie Hyperbolique et aux Surfaces de Riemann", Cassini, 2009.

[13] R. SCHOEN, Uniqueness, symmetry, and embeddedness of minimal surfaces, J. Differential Geom. 18 (1983), 791-809.

[14] M. SPIVAK, "A Comprehensive Introduction to Differential Geometry", Vol. IV. Third edition, Publish or Perish, Inc., Houston, Texas, 1999.

Dipartimento Ingegneria e Scienze dell'Informazione

e Matematica

Università di L'Aquila

via Vetoio - Loc. Coppito

67010 L'Aquila, Italia

nelli@univaq.it

Departamento de Matemática

Pontifícia Universidade Católica

do Rio de Janeiro

Rio de Janeiro

22453-900 RJ, Brazil

earp@mat.puc-rio.br

Université Paris Diderot, Paris 7

Institut de Mathématiques de Jussieu

UMR CNRS 7586

UFR de Mathématiques

Case 7012

Bâtiment Chevaleret

75205 Paris Cedex 13, France

toubiana@math.jussieu.fr 